\title{
Mathematical Model for During Ghrelin and Growth Hormone Serum Levels in Children
}

\author{
Geetha. $T^{1}$, Thangappan.R \\ ${ }^{1}$ Asst.Professor of Mathematics.K. N. Govt. Arts College for Women. Thanjavur. Tamilnadu. South India \\ ${ }^{2}$ Asst.Professor of Mathematics .Rajah serfoji. Govt. College . Thanjavur. Tamilnadu. South India
}

\begin{abstract}
In this paper we used Weighted Exponential distribution (WE) using the exponentiated class family of distributions. The proposed model named as Exponentiated Weighted Exponential distribution and it serves as an alternative to both the Weighted Exponential distribution and the Exponential distribution The exact role of ghrelin in the control of growth hormone $(G H)$ secretion has not been completely clarified as yet. Eighteen prepubertal children with short stature were included in the study. Using theresults of two GH-provocative tests (glucagon and clonidine), theparticipants were divided into two groups: GHD and NGH. In both groups, ghrelin levels were determined during the clonidine stimulation test .Different responses regarding ghrelin levels during the clonidine stimulation test were observed in the two study groups (GHD and NGH). A decrease in ghrelin levels was observed in the NGH children accompanied by a rise in the circulating GH levels, whereas the GHD children demonstrated a rise in both ghrelin and GH levels.
\end{abstract}

Keywords: Clonidine, Ghrelin, Growth hormone, Exponential Distribution, Reliability Analysis

\section{Application}

The effect of clonidine on ghrelin levels in short-stature children with or without growth hormone deficiency and uncover possible correlations of circulation GH with ghrelin throughout the clonidine test. No differences were observed in age, BMI z-score, height SDS and ghrelin levels between the two groups [1]. GH peak values were significantly higher and ghrelin peak values were lower in the NGH children compared to the GHD participants ( $\mathrm{p}=0.001$ and 0.013 , respectively) . Subjects with GHD demonstrated a significant

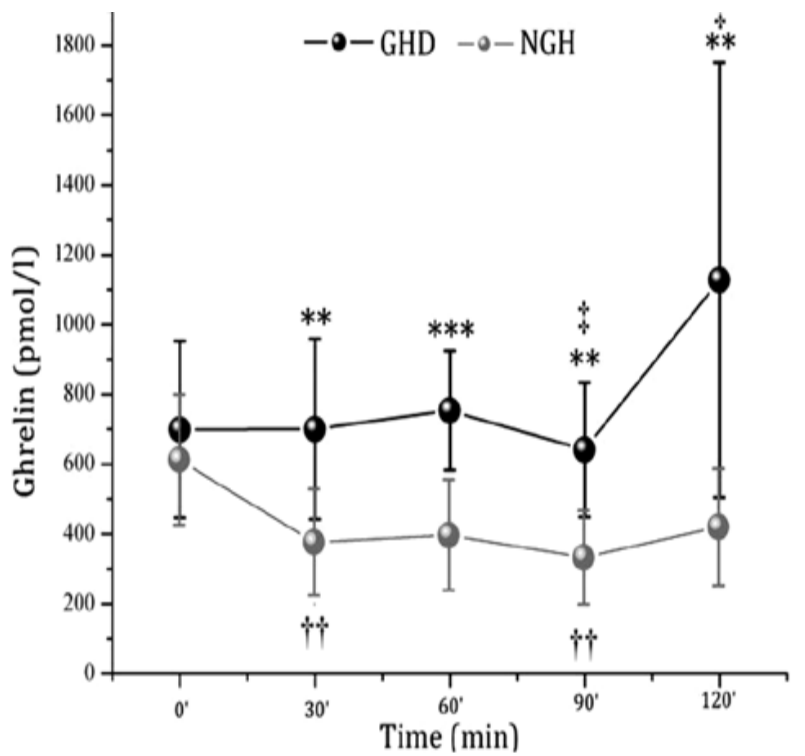

Figure 1. Mean \pm SD values of serum ghrelin during the clonidine test in the two groups. Significantly different from baseline using paired $t$-test $(\dagger \dagger \mathrm{p}=0.01, \uparrow \mathrm{p}=0.05)$. Significantly different from the previous time interval using paired t-test $(\$ \mathrm{p}=0.05)$. Significantly different from the NGH group using independent t-test $(* * \mathrm{p}=0.01$; $* * * \mathrm{p}=0.001)$.

fall in serum ghrelin concentration at $90 \mathrm{~min}$ and a rise at $120 \mathrm{~min}$ compared to the previous time interval (Figure 1). In the NGH group, circulating ghrelin was significantly lower at 30 min and 90 min compared to baseline ( $\mathrm{p}=0.009$ and 0.007 , respectively) [2]. Between the two studied groups, ghrelin levels were significantly higher in the GHD group at all time intervals Mean GH and ghrelin fluctuations within each group are demonstrated in Figure 2. GH concentration was higher in the NGH participants at 60 min and 90 min 
( $p=0.011$ and $p=0.001$, respectively) compared to the GHD children. GH levels significantly rose at $90 \mathrm{~min}$ $(\mathrm{p}=0.003)$ in the GHD participants and at all time intervals after $60 \mathrm{~min}$ in the NGH participants,compared to baseline an inverse relationship between ghrelin and $\mathrm{GH}$ in $\mathrm{NGH}$ children suggesting the existence of a $\mathrm{GH}-$ ghrelin feedback loop. Analogous interrelationship was not observed in the GHD children, indicating that the GH status possibly determines the relationship between circulating levels of ghrelin and GH [3]
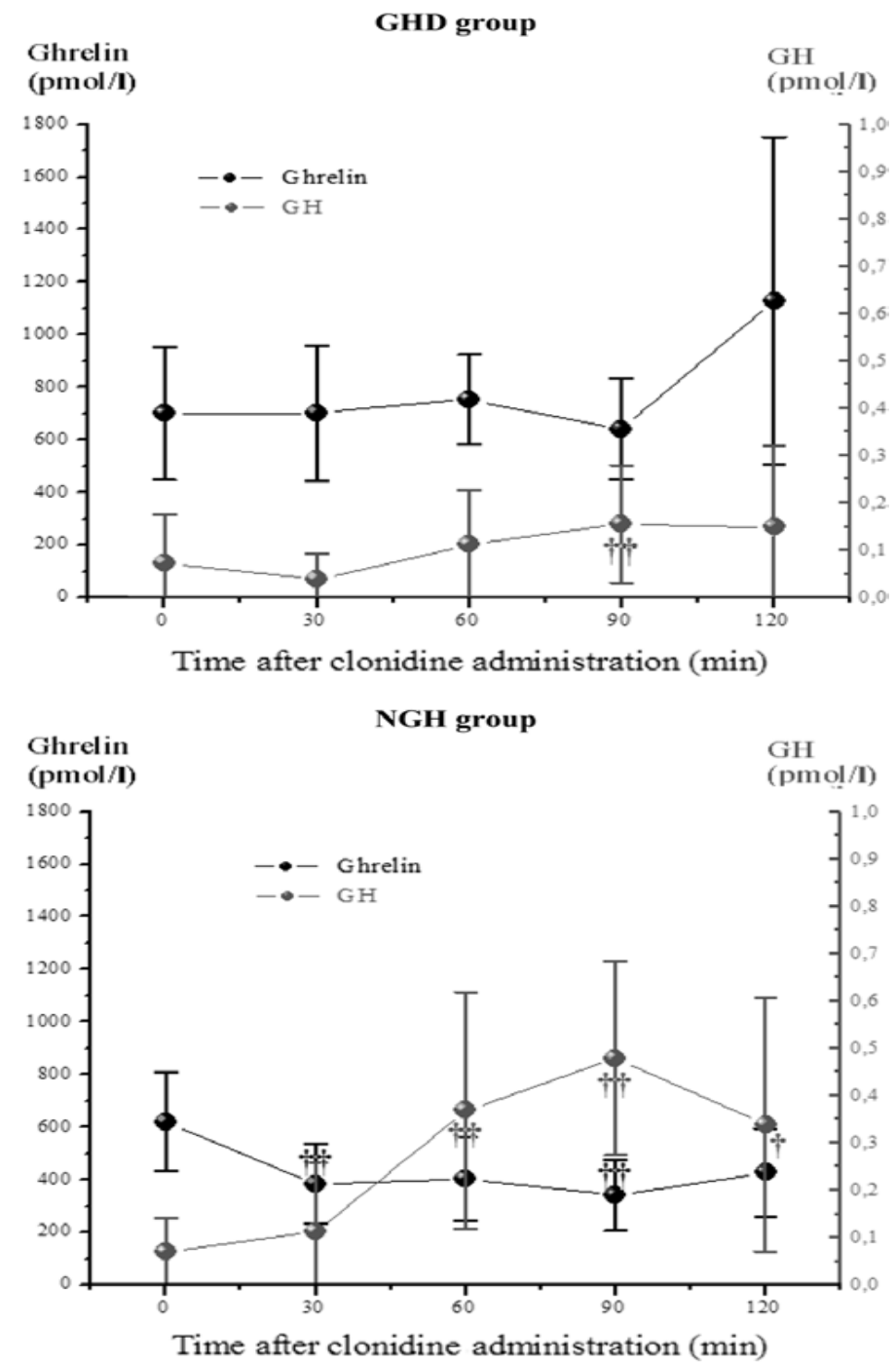

II. Mathematical Model

Extreme Value distributions have been found to be useful in the fields of engineering, insurance and modern science.Gupta and Kundu developed a two-parameter Weighted Exponential (WE) distribution as a lifetime model and it has been widely used engineering and medicine. In addition, theWEdistribution has been discovered to be a competitor tothe Weibull, Gamma and Generalized Exponential distributions. The probability density function (pdf) and the cumulative density function (cpftheWEdistribution are given respectively

$\mathrm{g}(\mathrm{x})=\{(\alpha+1) / \alpha\} \lambda \mathrm{e}^{-\lambda \mathrm{x}}\left[1-\mathrm{e}^{-\lambda \alpha \mathrm{x}}\right], x>0, \alpha>0, \lambda$

$\mathrm{G}(\mathrm{x})=[(\alpha+1) / \alpha]\left[1-\mathrm{e}^{-\lambda \mathrm{x}}-[1 /(1+\alpha)]\left\{\left(1-\mathrm{e}^{-\lambda \mathrm{x}(1+\alpha)}\right\}\right] ; x>0, \alpha>0, \lambda>0\right.$

where; $\square$ is the shape parameter $\square$ is the scale parameter

\section{The Exponentiated Weighted Exponential Distribution}

The Exponentiated family of distribution is derived by raising the cdf of an arbitrary parent distribution by a shape parameter say; $; \theta>0$. Its pdf is given by;

$f(x)=\theta(x) G(X)^{\theta-1}$

Its corresponding pdf is given by; 
$f(x)=g(x) G(x)^{\theta-1}$

With this understanding, we insert Equations (1) and (2) into Equation (3) to give the pdf of theExpWE as;

$f(x)=\theta\left(\quad[\alpha+1 / \alpha] \lambda e^{-\lambda x}\left[1-e^{-\lambda \alpha x}\right]\right)\left\{(\alpha+1 / \alpha)\left[1-e^{-\lambda x}-(1 / 1+\alpha)\left(1-e^{-\lambda x(1+\alpha)}\right)\right]\right\}^{\theta-1}$

The corresponding cdf of the ExpWE distribution is given by;

$\mathrm{F}(\mathrm{X})=\left\{[\alpha+1 / \alpha]\left[1-\mathrm{e}^{\lambda \mathrm{x}}(1 /(1+\alpha))\left(1-\mathrm{e}^{\lambda x(1+\alpha)]}\right\}\right.\right.$

For $x>0, \alpha>0, \lambda>0, \theta>0$

where; $\alpha$ and ${ }_{\theta}$ are the shape parameters $\lambda$ is the scale parameter [4]

\section{Reliability Analysis}

The reliability (or survival) function and the hazard function (or failure rate) of the proposed ExpWE distribution [5]

The survival function is mathematically represented by;

$S(x)=1-F(x)$

Therefore, the survival function of the ExpWE distribution is given by;

$\mathrm{S}(\mathrm{X})=1-\left\{\alpha+1 / \alpha\left[1-\mathrm{e}-^{\lambda \mathrm{x}} / 1+\alpha\left(1-\mathrm{e}^{\lambda \mathrm{x}}\right)\right]\right\} \theta$

Survival Function of the Exponentiated Weighted Exponential Distribution

The probability that a system having age ' $x$ ' units of time will survive up to ' $x \square t$ ' units of time for

$\mathrm{X}>0, \alpha>0, \lambda>0, \theta>0$

$\mathrm{S}(\mathrm{t} \mid \mathrm{x})=\mathrm{S}(\mathrm{x}+\mathrm{t}) / \mathrm{S}(\mathrm{x})$

$\mathrm{S} \mathrm{s}\left(\left.\mathrm{t}\right|_{\mathrm{x}}\right)=1-\left\{(\alpha+1 / \alpha)\left[1-\mathrm{e}^{-\lambda(\mathrm{x}+\mathrm{t})}-\left(1 /(1+\alpha)\left[1-\mathrm{e}^{-\lambda(\mathrm{x}+\mathrm{t})(1+\alpha)}\right]\right.\right.\right.$

$$
1-\left\{( \alpha + 1 / \alpha ) \left[1-\mathrm{e}^{-\lambda \mathrm{x}}-\left(1 /(1+\alpha)\left[1-\mathrm{e}^{-\lambda(\mathrm{x}+\mathrm{t})(1+\alpha)}\right\}^{\theta}\right.\right.\right.
$$

The hazard function is mathematically given by

$\mathrm{H}(\mathrm{x})=\mathrm{f}(\mathrm{x}) / 1-\mathrm{F}(\mathrm{x})$

Therefore, the expression for the hazard function of the ExpWE distribution is given by;

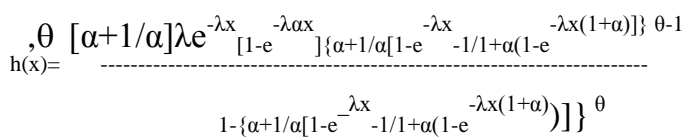

\section{Conclusion}

In this paper we found pdf and cdf of WE distribution for an inverse relationship between circulating gherlin and GH in NGH children, suggesting the presence of a negative feedback loop between gherlin and GH

\section{References}

[1] Kojima M, Hosoda H, Date Y, Nakazato M, Matsuo H, Kangawa K, 1999 Ghrelin is a growth hormone-releasingacylated peptide from stomach. Nature 402: 656-660

[2] Tschöp M, Smiley DL, Heiman ML, 2000 Ghrelin induces adiposity in rodents. Nature; 407: 908-913.

[3] Wren AM, Seal LJ, Cohen MA, et al, 2001 Ghrelin enhances appetite and increases food intake in humans.J Clin Endocrinol Metab 86: 5992-5995.

[4] AlexandraC, Cordeiro GM, sarabia JM,2012 Generallized Beta -Generalized Distributions Computational Statistics\&Data Analysis,56(6),1880-1897

[5] Andjelic G,Milosev 1,Djakovic v2010, Extreme Value Theory in Engineering Markets Economic Annals,LV(185),63-105 